\title{
Revisión: Cambio metabólico de las PAOs en el proceso de eliminación biológica y recuperación de fósforo.
}

\author{
Recibido 28 de octubre de 2016, Aceptado 16 de diciembre de 2016
}

Acevedo B. ${ }^{\mathbf{a}^{*}}$, Borrás $\mathbf{L}^{\mathrm{b}}$., Barat R. ${ }^{\mathrm{c}}$

${ }^{a}$ Universidad Autónoma de Nayarit. Ciudad de la Cultura "Amado Nervo" s/n, 63155. Nayarit, México.

${ }^{b}$ Departamento de Ingeniería Química, Universitat de València. Avda. de la Universidad, s/n. 46100 - Burjassot. Valencia, España. ${ }^{c}$ Instituto de Ingeniería del Agua y Medio Ambiente, Universitat Politècnica de València. Camino de Vera s/n, 46022. Valencia, España.

*E-mail: brenda.aj@gmail.com

Recibido 12 noviembre 2016, Aceptado 20 diciembre 2016

\section{Resumen}

Mediante tratamientos biológicos se puede efectuar la eliminación de nutrientes nitrógeno y fósforo de las aguas residuales. Las bacterias acumuladoras de polifosfatos (PAOs) son las responsables de la eliminación biológica de fósforo en condiciones anaerobias-aerobias/anóxicas. Estas bacterias compiten por el sustrato con las bacterias acumuladoras de glucógeno (GAOs). En estudios recientes se observó que cuando el contenido de polisfosfatos es bajo o casi nulo en las PAOs pueden comportarse metabólicamente como las GAOs. Este cambio de comportamiento puede ser aprovechado para la recuperación de fósforo de las aguas residuales.

Palabras clave: Eliminación biológica de fósforo; PAOs; GAOs; PAM; GAM; polifosfato; glucógeno.

\section{Introducci ón}

El fósforo y el nitrógeno son nutrientes que cuando rebasan los límites de depuración natural causan el grave problema de eutrofización en los cuerpos de aguas superficiales. Se considera un sistema eutrofizado cuando altos niveles de nutrientes principalmente nitrógeno y fósforo aceleran el crecimiento de organismos como plantas acuáticas y microorganismos fotosintéticos. Este crecimiento de organismos provoca cambios importantes en la calidad del agua de los sistemas ecológicos. El fósforo es considerado el nutriente más crítico, ya que en medios acuáticos continentales suele ser el reactivo limitante.

En el tratamiento biológico de aguas residuales, sobre los años 80 s se descubrió que en algunos fangos activos eran eliminadas grandes cantidades de fósforo del medio. Estos fangos eran transferidos de condiciones anaerobias a aerobias, los fangos resultantes contenían altas concentraciones de fósforo pudiendo ser empleados después de su deshidratación como fertilizantes. Amplias investigaciones sobre los procesos biológicos de eliminación de fósforo (EBPR) aportaron que los organismos responsables de llevar a cabo esta eliminación de fósforo son los llamados organismos o bacterias acumuladoras de polifosfatos (PAOs) $[1,2]$.

Las PAOs están dotadas con un comportamiento metabólico acumulador de polifosfato (PAM). Este metabolismo es observado cuando en condiciones anaerobias las bacterias son capaces de consumir ácidos grasos volátiles de cadena corta (AGV) como el ácido acético (Ac) [3-5]. Posteriormente, utilizan la adenosina de trifosfato (ATP) para transportar los AGV al interior de la célula y transformarlos en Acetil-CoA (Ac-CoA). El ATP necesario para el transporte de los AGV y su transformación a Ac-CoA es obtenido de la hidrólisis del polímero intracelular inorgánico polifosfato (poli-P) produciendo una liberación de fósforo soluble al medio. El Ac-CoA es transformado en polihidrixialcanoatos (PHA), para esto una fuente de reducción de equivalentes como la nicotinamida adenina dinucleótido (NADH) es requerida. Comeau y col. [6] así como Wentzel y col. [5] sugirieron que la obtención de NADH pudiera provenir del ciclo del ácido tricarboxilico (TCA), Mino y col. [4] de la degradación del glucógeno y, Pereira y col. [7] y Hesselmann y col. [8], de ambos. En condiciones aerobias, transforman el PHA en reservas de glucógeno y biomasa obteniendo energía para tomar el fósforo del medio en mayor cantidad que el fósforo soltado en condiciones anaerobias, e intracelularmente es transformado en poli-P, lo que produce una eliminación neta de fósforo del medio $[6,9]$.

En los procesos de EBPR intervienen diversas poblaciones de microorganismos que se encuentran mezclados e interrelacionados. Entre estos grupos se encuentran las bacterias acumuladoras de glucógeno (GAOs), las cuales, compiten con las PAOs por los AGV en condiciones anaerobias, pero sin contribuir a la suelta ni toma de fosfato del medio. Las GAOs tienen un metabolismo acumulador de glucógeno (GAM). En este metabolismo las bacterias toman los AGV del medio y los transforman en PHA, el ATP y NADH lo obtienen solo de la degradación del glucógeno. En condiciones aerobias, trasforman el PHA en glucógeno y biomasa [10]. Por lo tanto, cuando en el medio hay presencia de GAOs, estas compiten con las PAOs por los AGV del 
medio, reduciendo la disponibilidad del sustrato necesario para la eliminación biológica de fósforo. Esto permite el crecimiento de la población GAO y desplaza a la población de PAO del medio [11]. En consecuencia, se produce un deterioro del proceso de EBPR.

Liu $y$ col. [12] encontraron que un influente sintético con una relación baja de fósforo y materia orgánica (P/DQO) (ej. $<0.02 \mathrm{gP} / \mathrm{gDQO})$ podría estimular el crecimiento de las GAOs. Esto teóricamente se debería a la concentración insuficiente de fósforo para los requerimientos metabólicos de las PAOs, es decir, sería limitada la cantidad de poli-P intracelular y por lo tanto limitada la cantidad de energía para tomar los AGV del medio. Sin embargo, la utilización y recuperación del glucógeno es una parte integral del metabolismo de las PAOs. Por lo que en el 2003, Schuler y Jenkins [13] sostuvieron la hipótesis de que las PAOs pudieran presentar un GAM cuándo el fósforo es insuficiente.

Estudios recientes realizados por Barat y col. [14] obtuvieron que en un sistema con eliminación biológica de fósforo, las PAOs presentaron un cambio metabólico hacia el GAM cuando se reducía la cantidad de fósforo disponible debido a procesos de precipitación como fosfato cálcico. Por otro lado, Zhou y col. [15] también observaron un cambio en el comportamiento metabólico en las PAOs, al reducir directamente la concentración de fósforo en el agua del influente.

En los últimos años se llevaron a cabo estudios sobre el metabolismo de las PAOs cuando la biomasa presenta distintos contenidos de poli-P [16,17]. La finalidad de este escrito es difundir el conocimiento sobre el comportamiento metabólico de las PAOs cuando estas carecen de poli-P en los procesos EBPR para el tratamiento de aguas residuales, y como esta versatilidad podría contribuir a la recuperación de $\mathrm{P}$.

\section{Metabolismo de las PAOs cuando el contenido en poli-P es bajo.}

Las PAOs y GAOs son microorganismos muy parecidos metabólicamente por lo que algunos autores llegaron a pensar que tal vez las PAOs y las GAOs pudieran ser el mismo microorganismo. La única diferencia entre estos microorganismos que había sido observada había sido que las PAOs degradaban sus reservas intracelulares de poli-P en condiciones anaerobias provocando una liberación de fosfatos al medio, y en condiciones aerobias tomaban el fósforo del medio regenerando el poli-P intracelular. Mientras que en medios con GAOs no había una suelta de fósforo, solo se observaba un consumo de los AGV y los almacenan en grandes cantidades de glucógeno [18-20].

Más tarde, varios estudios microbiológicos mediante la técnica de PCR (reacción en cadena de la polimerasa) y FISH (hibridación con fluorescencia in situ) revelaron que los microorganismos PAOs y GAOs son distintos taxonómica como morfológicamente [2,21,22], tal y como se observa en la figura 1.

Por otra parte, se encontraron indicios de que las PAOs y las GAOs usan rutas metabólicas similares $[13,23]$. Estudios realizados con $\mathrm{C} 13$ concluyeron que las PAOs utilizaban parte del TCA en condiciones anaerobias [24]. En los estudios realizados por Pereira $y$ $\mathrm{col}$. [7] y Zeng y col. [25] entre otros, sugirieron que en condiciones aerobias o anóxicas tanto las PAOs como las GAOs, empleaban el ciclo del TCA y la cadena respiratoria para la obtención de energía y poder reductor. En el caso de las PAOs la energía la empleaban para capturar los fosfatos del medio y almacenarlos como poli-P intracelular; y tanto las PAOs como las GAOs empleaban el poder reductor y energía para la síntesis de glucógeno, el crecimiento y mantenimiento celular a partir de las reservas de PHA. Zhou y col.[15] proponen que las GAOs podrían emplear la rutas metabólicas anaerobias mostradas en la figura 2. Estas rutas metabólicas sugieren que el ATP y NADH necesario para la toma de $\mathrm{AGV}$ del medio provienen de la degradación del glucógeno. Y el carbono es almacenado como PHA en diferentes unidades monoméricas de $\mathrm{PHV}, \mathrm{PH} 2 \mathrm{MB}$ y $\mathrm{PH} 2 \mathrm{MV}$

En trabajos recientes de Acevedo y col. [17,26] estudiaron en condiciones anaerobias/aerobias el comportamiento metabólico de las PAOs. Su experimentación consistió en la reducción a diferentes niveles del contenido de poli-P intracelular de los organismos y mantenerlos con una relación de $\mathrm{P} / \mathrm{DQO}$ menor a $0.02 \mathrm{gP} / \mathrm{gDQO}$ en el influente, por un largo plazo (3 TRC). En estos experimentos observaron el proceso de toma de AGV y la evolución de los polímeros intracelulares glucógeno y PHA. Así como la población bacteriana a corto y largo plazo con bajos contenidos en poli-P intracelular.

Los resultados de estos estudios mostraron que a pesar de la reducción del poli-P intracelular de la biomasa se presentaba una toma de AGV del medio, y una degradación considerable del glucógeno cuando el contenido en poli-P intracelular era casi nulo. Así mismo, se observó una mayor producción de PHA al reducir el contenido de poli-P. Los datos generados a corto como a largo plazo sugieren que las PAOs presentaron un cambio metabólico de PAM a GAM al extraer el poli-P intracelular. Los resultados microbiológicos, incluso al someter la población de PAOs con una relación baja de $\mathrm{P} / \mathrm{DQO}$ menor a $0.02 \mathrm{gP} / \mathrm{gDQO}$, indicaron que no se presentó un cambio ni desplazamiento de la población de PAOs. Es preciso señalar que en estos experimentos las condiciones operacionales favorecían en todo momento a las PAOs, siendo la cantidad de bacterias GAOs despreciable en el sistema durante toda la fase experimental. Por lo tanto, concluyeron que cuando hay bajos contenidos de fósforo en el sistema la PAOs pueden adaptarse a un GAM y ser ellas y no las GAOs las que estén presente en el sistema.

A partir de las observaciones en Acevedo y col. $[17,26]$ los autores propusieron que las PAOs emplean 
las rutas metabólicas anaerobias mostradas en la figura 3. Las rutas sugieren que el HAc consumido es transformado en acetil-CoA. El acetil-CoA puede seguir dos rutas de acuerdo a las necesidades: la ruta de

\section{Hibridación FISH: PAOmix/Eubmix, 630x.}

Clase: $\beta$-Proteobacteria

\section{Hibridación FISH: GAOmix+DF1+DF2/Eubmix, 630x \\ Clase: $\gamma$-Proteobacteria y a-Proteobacteria}

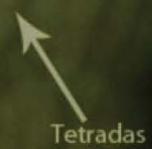

Figura 1. Morfología de las PAOs y GAOs hibridadas con sondas específicas de FISH. Muestra obtenida de un cultivo enriquecido en PAOs $(93 \pm 1.9 \%)$ y GAOs $(1 \pm 0.6 \%)[16,17]$.

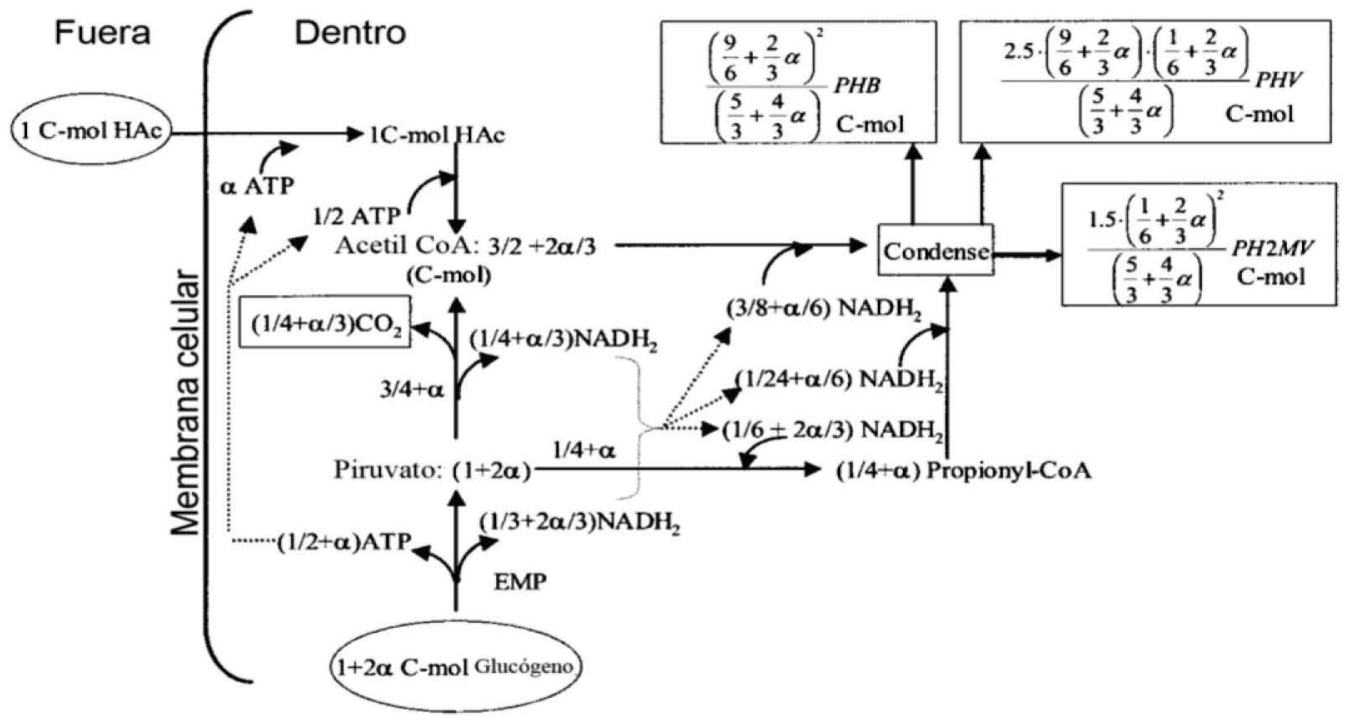

Fig 2.2. Metabolismo anaerobio de las GAOs [15]. 


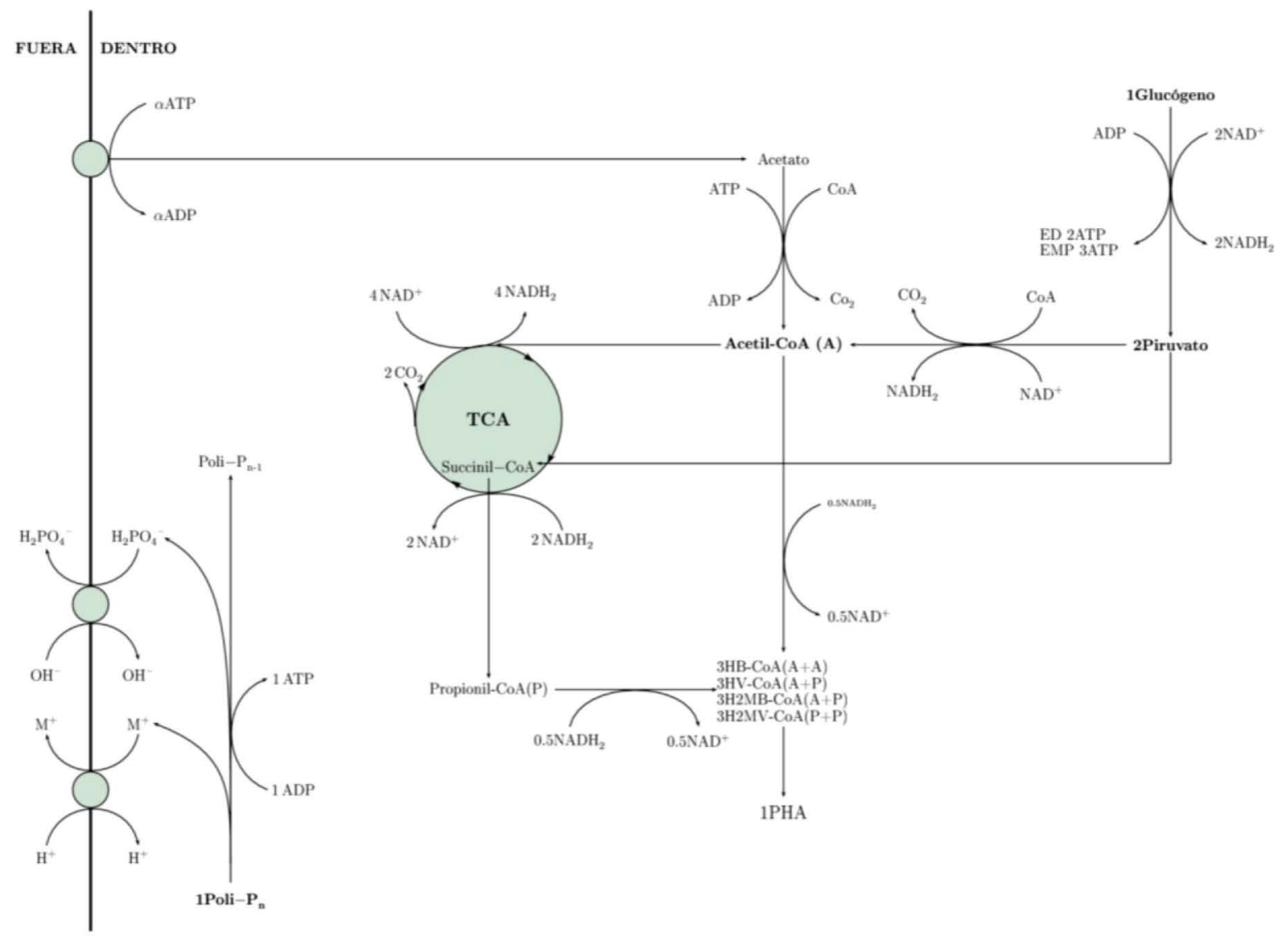

Fig. 2.3 Esquema metabólico anaerobio para las PAOs $[8,16,23,24,27]$. 
unión de unidades monoméricas acetil-acetil para la formación de PHB y se consumen NADH, o la ruta del TCA donde se generaría NADH y produce succinil-CoA que posteriormente se transforma en propionil-CoA con consumo de NADH y se forman unidades monomericas acetil-propionil (PHV). Las PAOs en presencia de poli-P, utilizan principalmente la glucólisis para la producción de NADH, ya que el ATP necesario para la toma de AGV y el mantenimiento celular anaerobio lo obtienen de la hidrólisis del poli-P. Sin embargo, cuando el contenido en poli-P es bajo, tiene que emplearse la ruta glucolítica con el fin de compensar la falta de ATP. Es posible que el uso de la vía glucolítica para abastecer las necesidades de ATP genere un exceso de NADH, entonces el piruvato resultante de la glucólisis podría tomar dos rutas: la formación de acetil-CoA empleada en menor medida, ya que generaría más $\mathrm{NADH}$; la síntesis succinil-pro-pionil CoA emplea principalmente la ruta parcial del TCA equilibrando el NADH en el interior de la célula. La ruta piruvato-succinil-propionil CoA podría ser potenciada al ser inhibida la enzima dehidrogenasa succinato del ciclo del TCA provocando que el NADH no sea reoxidado, esto provocaría una acumulación de succinil-CoA la cual sería incorporada a la ruta succinilpropionilCoA para la formación de unidades monoméricas de PHV, PH2MB y PH2MV [13].

En base a los estudios de Barat y col. [14-16,26], cuando las PAOs presentan un metabolismo PAM podrían emplear la hidrólisis de poli-P y en menor proporción la degradación del glucógeno para la obtención de energía, para la generación de poder reductor estos organismos podrían emplear la glucólisis como ruta metabólica. El ciclo del TCA lo podrían emplear para incorporar el acetil-CoA y sintetizar unidades monoméricas principalmente PHB y en menor cantidad PHV. Cuando las PAOs tienen bajos o casi nulos contenidos de poli-P podrían emplear la glucólisis generando energía para la toma de $\mathrm{AGV}$ y poder reductor para la síntesis de PHA, potenciar la ruta parcial del TCA piruvato-succinil-propionil $\mathrm{CoA}$ proporcionando equilibrio energético en el interior de la célula y potenciando la formación de unidades monoméricas de PHV, PH2MB y PH2MV, presentando así un metabolismo GAM.

\section{Recuperaci ón de $\mathbf{P}$}

Este cambio de metabolismo proporciona la oportunidad de recuperar fósforo de una forma sencilla y económica.

Es bien conocido que el fósforo es un componente esencial para la vida, debido a que desempeña un papel crucial en los procesos biológicos. Su principal uso radica en la industria de los fertilizantes en forma de fosfatos, procedentes de la extracción en las rocas fosfáticas. Según apuntan determinados estudios, las reservas naturales de fósforo serán incapaces de aportar el fósforo necesario demandado a nivel mundial en los próximo 50 a 100 años, por la sobreexplotación de dicho mineral [28]. De hecho, en la actualidad se ha observado una disminución en la calidad de las reservas, elevando el coste de su extracción, procesamiento y transporte.

En este sentido, basado en los estudios de Cordell $y$ col. [29] aproximadamente el $17 \%$ del fósforo procedente de la extracción de las rocas fosfáticas termina en las aguas residuales, que en caso de ser vertidas sin tratamiento previo ocasionan el grave problema de contaminación en masas de agua, conocido como eutrofización. Por todo ello, es importante no solo eliminar el fósforo del agua residual, sino también su posterior recuperación como fertilizante.

Actualmente existen varios procesos para la recuperación de fósforo de las aguas residuales en las plantas de tratamiento. Estos procesos suelen emplearse sobre la línea de lodos y la línea de agua de las plantas de tratamiento de aguas residuales.

Sobre la línea de lodos, los métodos empleados según Yuan y col. [30] son:

a) La aplicación directa de los biosólidos deshidratados al suelo.

b) La liberación de $\mathrm{P}$ de lodos del proceso de eliminación biológica de fósforo (EBPR) por métodos biológicos seguidos de recuperación mediante métodos químicos.

c) La liberación de $\mathrm{P}$ de los lodos EBPR por métodos térmico-químicos, seguido de la utilización de residuos o de procesamiento adicional para su recuperación.

Sin embargo, estos métodos conlleva algunas desventajas como no poder evitar una precipitación incontrolada en la unidad de digestión y la corrosión de los dispositivos de gestión de lodos aguas abajo debido a una importante liberación de $\mathrm{P}$ y aumento de $\mathrm{pH}$ durante la digestión [31].

De la línea de agua, las configuraciones más antiguas son: Phostrip [32] y BCFS [33], las cuales requieren dos unidades de operación y la concentración en la corriente no es lo suficientemente alta como para asegurar una alta eficiencia de recuperación de $\mathrm{P}$ en el proceso de cristalización posterior (alrededor de $25 \mu \mathrm{g} / 1$ en la fase anaeróbica [34]).

En el estudio reciente de Acevedo y col. [17] proponen una metodología para la recuperación de $\mathrm{P}$, operando un sistema SBR. La metodología es basada en emplear la capacidad que las PAOs tienen para cambiar su metabolismo y extraer severamente el $\mathrm{P}$ acumulado para obtener una corriente de fósforo altamente concentrada, sin que el sistema sea deteriorado. Según sus estudios, está metodología puede recuperar hasta un $81 \%$ del fósforo del agua residual.

Otros estudios sobre la viabilidad de la recuperación de $\mathrm{P}$ de una corriente enriquecida en la línea de agua usando diferentes configuraciones son los realizados por Hiroya y col. [35], Shi y col. [36], Wong y col. [37] y Xia $y$ col. [38]. 


\section{Conclusi ones}

Las PAOs son los organismos responsables de la eliminación biológica de fósforo. Estos organismos tienen la capacidad de bioacumular el fósforo como poli$\mathrm{P}$ intracelular y de cambiar su metabolismo acumulador de fósforo a un metabolismo acumulador de glucógeno cuando el contenido en poli-P intracelular es bajo o casi nulo. La recuperación de fósforo proveniente de las aguas residuales es importante. La capacidad de las PAOs al cambiar su metabolismo ofrece una metodología eficiente de obtener una corriente altamente enriquecida mediante un sistema sencillo SBR para recuperar el fósforo desde la línea de agua en el tratamiento de aguas residuales.

\section{Referencias}

1. Crocetti, G. R.; Hugenholtz, P.; Bond, P. L.; Schuler, A.; Keller, J.; Jenkins, D.; Blackall, L. L. Appl. Environ. Microbiol. 2000, 66, 1175-1182.

2. Hesselmann, R. P. X.; Werlen, C.; Hahn, D.; van der Meer, J. R.; Zehnder, A. J. B. Syst. Appl. Microbiol. 1999, 22, 454-465.

3. Comeau, Y.; Hall, K. J.; Oldham, W. K. Apply Enviromental Microbiol. 1988, 54, 2325-2327.

4. Mino, T.; Arun, V.; Tsuzuki, Y.; Matsuo, T. Advances in Water Pollution Control: Biological Phosphate Removal from Wastewater; Pergamon Press: Oxford, 1987; pp 2738 .

5. Wentzel, M. C.; Lotter, L. H.; Loewenthal, R. E.; Marais, G. v. R. Water SA 1986, 12, 209-224.

6. Comeau, Y.; Hall, K. J.; Hancock, R. E. W.; Oldham, W. K. Water Res. 1986, 20, 1511-1521.

7. Pereira, H.; Lemos, P. C.; Reis, M. A. M.; Crespo, J. P. S G.; Carrondo, M. J. T.; Santos, H. Water Res. 1996, 30 (9), $2128-2138$.

8. Hesselmann, R. P. X.; Rummell, R. V.; Resnickm, S. M.; Hany, R.; Zehnder, A. J. B. Water Res. 2000, 34, 34873494.

9. Wentzel, M. C.; Dold, P. L.; Ekama, G. A.; Marais, G. v. R. Water A 1989, 15 (2), 89-102.

10. Liu, W.-T.; Mino, T.; Matsuo, T.; Nakamura, K. Water Sci. Technol. 1996, 34 (1-2), 25-32.

11. Cech, J. S.; Hartman, P. Water Res. 1993, 27, 1219-1225.

12. Liu, W.-T.; Nakamura, K.; Matsuo, T.; Mino, T. Water Res 1997, 31 (6), 1430-1438

13. Schuler, A. J.; Jenkins, D. Water Environ. Res. 2003, 75 (6), 485-498.

14. Barat, R.; Montoya, T.; Borrás, L.; Ferrer, J.; Seco, A Water Res. 2008, 42 (13), 3415-3424.

15. Zhou, Y.; Pijuan, M.; Zeng, R. J.; Huabing, L.; Yuan, Z. Water Res. 2008, 42, 2361-2368.

16. Acevedo, B.; Oehmen, A.; Carvalho, G.; Seco, A.; Borrás, L.; Barat, R. Water Res. 2012, 46 (6), 1889-1900.
17. Acevedo, B.; Camiña, C.; Corona, J. E.; Borrás, L.; Barat, R. Chem. Eng. J. 2015, 270, 459-467.

18. Mino, T.; van Loosdrecht, M. C. M.; Heijnen, J. J. Water Res. 1998, 32 (11), 3193-3207.

19. Bond, P. L.; Erhart, R.; Wagner, M.; Keller, J.; Blackall, L. L. Appl. Environ. Microbiol. 1999, 65 (9), 4077-4084.

20. Jeon, C. O.; Lee, D. S.; Lee, M. W.; Park, J. M. Water Environ. Res. 2001, 73, 301-306.

21. Nielsen, A. T.; Liu, W. T.; Filipe, C.; Grady, C.; Molin, S.; Stahl, D. A. Appl. Environ. Microbiol. 1999, 65, 12511258.

22. Welles, L.; Tian, W. D.; Saad, S.; Abbas, B.; LopezVazquez, C. M.; Hooijmans, C. M.; van Loosdrecht, M. C. ; Brdjanovic, D. Water Res. 2015, 83, 354-366.

23. Reis, M. A. M.; Serafim, L. S.; Lemos, P. C.; Ramos, A. M.; Aguiar, F. R.; van Loosdrecht, M. C. M. Bioprocess Biosyst. Eng. 2003, 25 (6), 377-385.

24. Martín, H. G.; Ivanova, N.; Kunin, V.; Warnecke, F.; Barry, K. W.; McHardy, C., A. C.an.Yeates; He, S.; Salamov, A. A.; Szeto, E.; Dalin, E.; Putnam, N. H.; Shapiro, H. J.; J.L, P.; Rigoutsos, I.; Kyrpides, N. C.; Blackall, L. L.; McMahon, K. D.; Hugenholtz, P. Nat. Biotechnol. 2006, 24, 1263-1269.

25. Zeng, R. J.; Yuan, Z.; Keller, J. Biotechnol. Bioeng. 2003, 83 (3), 293-302.

26. Acevedo, B.; Murgui, M.; Borrás, L.; Barat, R. Chem. Eng. J. 2017, 311, 82-90.

27. Zhou, Y.; Pijuan, M.; Zeng, R. J.; Yuan, Z. Water Res. 2009, 43 (5), 1330-1340.

28. Cordell, D.; Drangert, J. O.; White, S. Glob. Environ Change 2009, 19 (2), 292-305.

29. Cordell, D.; Rosemarin, A.; Schröder, J. J.; Smit, A. L. Chemosphere 2011, 84 (6), 747-758.

30. Yuan, Z.; Pratt, S.; Batstone, D. J. Curr. Opin. Biotechnol. 2012, $23(6), 878-883$.

31. Doyle, J. D.; Parsons, S. A. Water Res. 2002, 36 (16) $3925-3940$

32. Levin, G. V.; Della Sala, U. In Biological Phosphate Removal from Wastewaters; Pergamon Press Oxford, 1987; pp 249-259.

33. van Loosdrecht, M. C. M.; Brandse, F. A.; De Vries, A. C. Water Sci. Technol. 1998, 37 (9), 209-217.

34. Barat, R.; Montoya, T.; Borrás, L.; Seco, A.; Ferrer, J. Water Sci. Technol. 2006, 53 (12), 29-37.

35. Hiroya, K.; Masashi, H.; A, K.; K, T.; O, N.; O, A. Water Res. 2013, 47 (6), 2025-2032.

36. Shi, J.; Lu, X.; Yu, R.; Zhu, W. Bioresour. Technol. 2012, $121(0), 183-189$

37. Wong, P. Y.; Cheng, K. Y.; Kaksonen, A. H.; Sutton, D. C.; Ginige, M. P. Water Res. 2013, 47 (17), 6488-6495.

38. Xia, C.-W.; Ma, Y.-J.; Zhang, F.; Lu, Y.-Z.; Zeng, R. J. Appl. Biochem. Biotechnol. 2014, 172 (2), 820-828. 